\title{
Capsule endoscopy in inflammatory bowel disease: when and how
}

\author{
Ida Hilmi ${ }^{1}$, Taku Kobayashi ${ }^{2}$ \\ ${ }^{1}$ Department of Medicine, University of Malaya Medical Centre, Kuala Lumpur, Malaysia; ${ }^{2}$ Center for Advanced IBD Research and Treatment, \\ Kitasato University Kitasato Institute Hospital, Tokyo, Japan
}

Capsule endoscopy (CE) is emerging as an important investigation in inflammatory bowel disease (IBD); common types include the standard small bowel CE and colon CE. More recently, the pan-enteric CE was developed to assess the large and small bowel in patients with Crohn's disease (CD). Emerging indications include noninvasive assessment for mucosal healing (both in the small bowel and the colon) and detection of postoperative recurrence in patients with CD. Given the increasing adoption, several CE scoring systems have been specifically developed for IBD. The greatest concern with performing CE, particularly in $\mathrm{CD}$, is capsule retention, but this can be overcome by performing cross-sectional imaging such as magnetic resonance enterography and using patency capsules before performing the procedure. The development of software for automated detection of mucosal abnormalities typically seen in IBD may further increase its adoption. (Intest Res 2020;18:265-274)

Key Words: Capsule endoscopy; Crohn disease; Colitis, ulcerative; Inflammatory bowel disease

\section{INTRODUCTION}

Since its introduction, capsule endoscopy (CE) has revolutionized the imaging of the small bowel. Previously, endoscopic images of the whole length of the small bowel were generally not attainable; short-segment images using push enteroscopy with a colonoscope or on-table enteroscopy during a laparotomy were used. However, in 2000, Iddan et al. ${ }^{1}$ introduced the first wireless CE for visualizing the whole length of the small bowel. Since then, its role has expanded as an important noninvasive method for visualizing the rest of the gastrointestinal tract. The main indication for CE is the investigation of obscure gastrointestinal bleeding, but it has been increasingly used for assessing inflammatory bowel disease (IBD); mainly Crohn's disease (CD), but also ulcerative colitis (UC).

This review summarizes the current status of CE in IBD,

Received December 9, 2019. Revised May 3, 2020. Accepted May 26, 2020. Correspondence to Ida Hilmi, Department of Medicine, University of Malaya Medical Centre, Jalan Lembah Pantai, Kuala Lumpur 59100, Malaysia. Tel: +60-3-79492965, Fax: +60-3-79492966, E-mail: ida.hilmi@gmail.com with a focus on emerging indications and new developments that may enhance its future use.

\section{TYPES OF CAPSULE ENDOSCOPY}

The original CE, the PillCam (Fig. 1A), was manufactured by Given Imaging Ltd. (Yokne'am Illit, Israel). This has been followed by the development of several other capsules by other manufacturers, which include MiroCam (Intromedic, Seoul, Korea) (Fig. 1B), EndoCapsule (Olympus, Tokyo, Japan), OMOM (Jianshan Science and Technology [Group] Co., Ltd, Chongqing, China; CapsoCam, Capsovision, Saratoga, CA, USA).

As mentioned previously, the original CEs were designed for visualization of the small bowel, which was notoriously inaccessible to the endoscopist due to its length and tortuosity. Since the original small bowel CE (SBCE) was introduced, it has undergone many developments such as enhanced image resolution, faster adaptable frame rate, and capability for realtime analysis to further increase the diagnostic yield.

Subsequently, the colon CE (CCE) was developed in 2006 
to allow noninvasive visualization of the colon. ${ }^{2}$ The CCE-2, such as the PillCam COLON 2 (Fig. 1C), has enabled imaging which is superior to that of the first generation CCE. More recently, the PillCam Crohn's Capsule (PCC) (Medtronic) was developed, which is a pan-enteric video capsule system that allows visualization of the small and the large bowel. ${ }^{3}$

\section{INDICATIONS FOR CAPSULE ENDOSCOPY IN IBD}

Almost in parallel with the development of CE, device-assisted enteroscopy (DAE), which may be balloon-assisted or spiral,
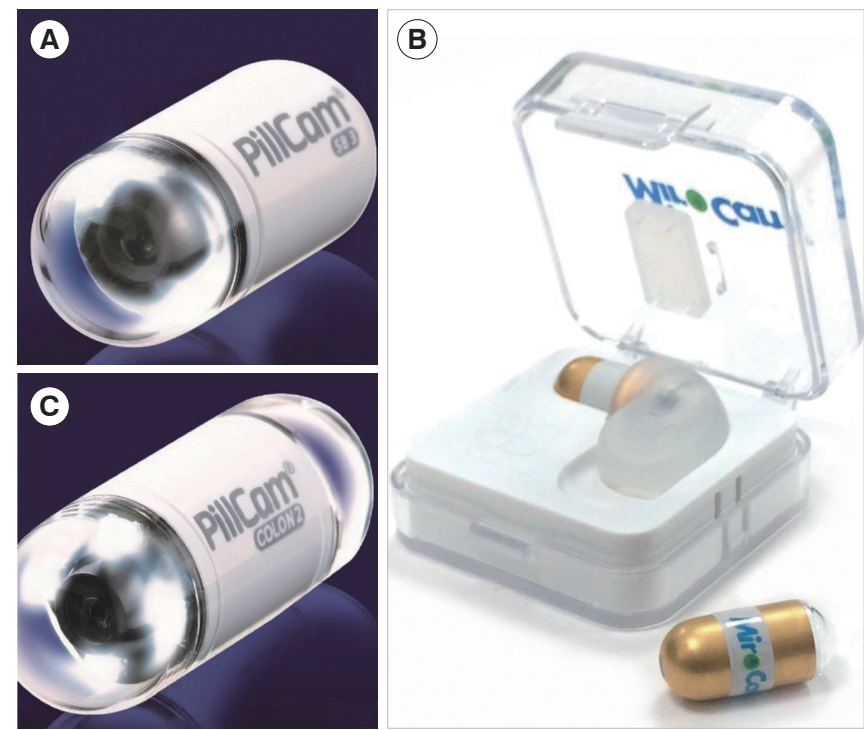

Fig. 1. Types of capsule endoscopy. (A) PillCam Small Bowel Capsule 3 (SB3; Given Imaging Ltd.), (B) MiroCam capsule (Intromedic), and (C) PillCam Colon Capsule Endoscopy 2 (Given Imaging Ltd.). was introduced with the added advantage of tissue acquisition and therapeutic procedures. $\mathrm{CE}, \mathrm{DAE}$, and magnetic resonance enterography (MRE) are generally preferred for small bowel visualization; they may be used alone or in combination depending on the clinical situation (see the suggested algorithm for suspected or established CD).

The indications for IBD are as follows: (1) to investigate patients when there is a high index of suspicion for CD, but esophagogastroduodenoscopy and ileocolonoscopy findings are normal; (2) to assess small bowel involvement or disease activity in patients with confirmed CD; (3) to assess postoperative recurrence of $\mathrm{CD}$ in the small bowel; or (4) to monitor disease activity or mucosal healing of the colon in UC (and colonic CD).

\section{To Investigate Patients When There Is a High Index of Suspicion for Isolated Small Bowel CD}

Up to $30 \%$ of CD patients have isolated small bowel disease, ${ }^{4}$ and CE plays an important role in the diagnosis in these cases. These patients were historically very difficult to diagnose as they could initially present with nonspecific symptoms (mild abdominal pain with or without diarrhea) and get labeled as functional given the initial negative investigations. A large meta-analysis revealed that CE was far superior to small-bowel radiography ( $52 \%$ vs. 16\%), computed tomography enterography (CTE; $68 \%$ vs. $21 \%$ ), and ileocolonoscopy ( $47 \%$ vs. $25 \%$ ), but not MRE in the diagnosis of suspected non-stricturing small-bowel CD. ${ }^{5}$ Another meta-analysis also showed that there were no differences in the diagnostic yield of CE and MRE and small bowel contrast ultrasound. ${ }^{6}$ In contrast, sever-
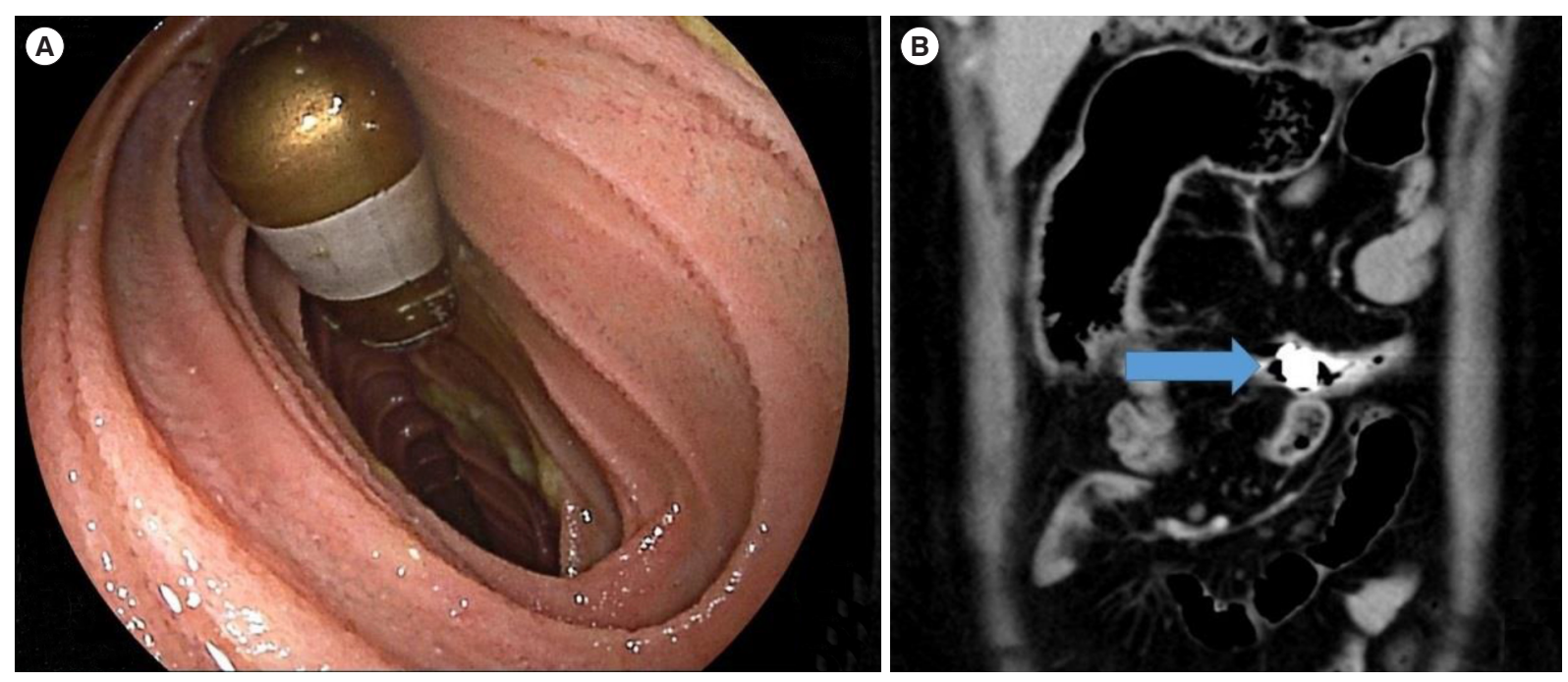

Fig. 2. Endoscopic (A) and computed tomography (B) images of a retained capsule. 
al other studies have shown that CE was superior to MRE in detecting subtle, early lesions. ${ }^{7}$ Consequently, the role of CE in suspected isolated small bowel CD has been long established and incorporated into most recommendations and consensus guidelines. $^{8-10}$

However, the main concern in using CE in patients with suspected $\mathrm{CD}$ is the risk of capsule retention (Fig. 2). In gener$\mathrm{al}$, the risk of retention is low, but this is increased up to $3.6 \%$ in patients with suspected CD alone and up to $10.4 \%$ in suspected and definite CD combined. ${ }^{11-14}$ Therefore it is not advisable to carry out CE unless imaging such as MRE has been avoid repetition carried out first. In addition, it is recommended that patency capsules (PCs), which are dissolvable radioopaque capsules (Fig. 3), should also be administered before the actual CE. ${ }^{15}$ The use of PCs rules out small bowel obstruction in about $99 \%$ of cases, but it does not exclude it altogether. ${ }^{16}$ The retention of PCs has been reported to result in abdominal pain and, in a few cases, complete small bowel obstruction and perforation. PCs also add to the cost of the overall procedure.

Fortunately, most cases of retention of the capsule are asymptomatic. A trial of steroids is often administered to reduce inflammation and facilitate the egestion of the capsule. If this is unsuccessful, most capsules can safely be removed by DAE. Approximately, $32 \%$ to $45 \%$ of cases will require surgery. 17,18

While it is important to make a timely diagnosis of IBD, it is equally important not to make an erroneous diagnosis of IBD. First of all, many differential diagnoses exist, such as NSAID induced enteropathy, intestinal Behçet's disease, and lymphoma, which may have a similar presentation to IBD endoscopically. Therefore, any significant findings on CE must be followed-up with enteroscopy and biopsies. Secondly, small bowel breaks in mucosa/lesions are common and asymptomatic, and they may lead to an over-diagnosis of IBD. The Lewis score (LS) was developed to differentiate between significant and nonsignificant inflammation of the bowel, as well as categorize the degree of inflammation (Table 1) ${ }^{19}$ The categories based on cutoff values are as follows; a score of $<135$ is considered normal or clinically insignificant, a score between 135 and 790 is mild, and a score of $\geq 790$ is moderate to severe. In a study looking at the diagnostic yield of the LS for suspected CD; the cutoff score of $\geq 135$ had an overall diagnostic accuracy of $83.2 \%$ with sensitivity, specificity, positive predictive value, and negative predictive value of $89.5 \%, 78.9 \%, 73.9 \%$, and $91.8 \%$, respectively. ${ }^{20}$

\section{To Assess Small Bowel Involvement or Disease Activity in Patients with Confirmed CD}

It is accepted that all cases of CD should undergo a type of small bowel imaging, and MRE has been the most widely utilized. MRE allows transmural visualization of the small bowel, a more precise anatomical "roadmap," and the identification of any concurrent abdominal abscesses. Although most guidelines have not recommended routine CE for established CD with normal MRE or $\mathrm{CTE},{ }^{10}$ it can be considered in certain situations such as unexplained anemia, severe malnutrition, and inconsistency between symptoms and other imaging findings. A study by Dubcenco et al. ${ }^{21}$ showed that active disease was identified by CE, ileocolonoscopy, and barium radiography in $82 \%, 49 \%$, and $32 \%$ of symptomatic patients. In another similar study, the findings from CE led to the escalation of treatment in $45 \%$ of these patients. ${ }^{22}$

Endoscopic mucosal healing is now considered an important target in the management of IBD, even in asymptomatic patients. For example, Ben-Horin et al. ${ }^{23}$ found that endoscopic
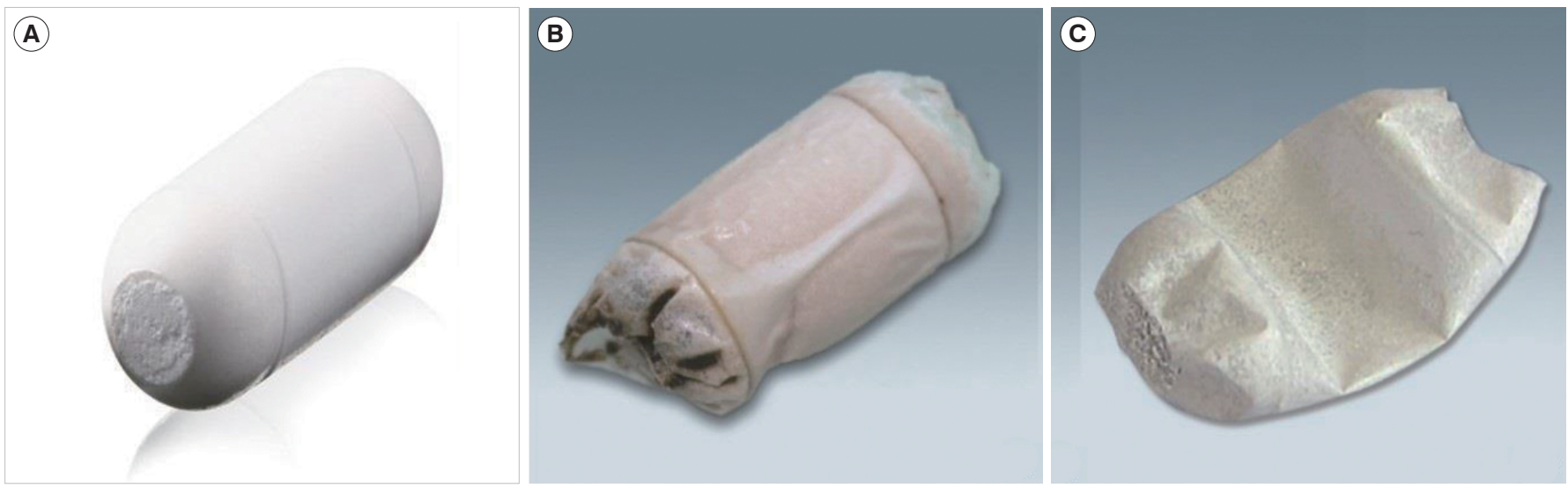

Fig. 3. Patency capsules (A) intact, (B) partially dissolved, and (C) fully dissolved. 
Table 1. The Lewis Score for the Assessment of Small Bowel Lesions Using Small Bowel Capsule Endoscopy

\begin{tabular}{|c|c|c|c|}
\hline Parameters & Number & Longitudinal extent & Descriptors \\
\hline \multicolumn{4}{|l|}{ First tertile } \\
\hline \multirow[t]{2}{*}{ Villous appearance } & Normal - 0 & Short segment - 8 & Single - 1 \\
\hline & & Whole tertile - 20 & Diffuse -17 \\
\hline \multirow[t]{3}{*}{ Ulcer } & None - 0 & Short segment - 5 & $<1 / 4-9$ \\
\hline & Few - 5 & Whole tertile - 15 & $>1 / 2-18$ \\
\hline & Multiple - 10 & & \\
\hline \multicolumn{4}{|l|}{ Second tertile } \\
\hline Villous appearance & Normal - 0 & Short segment - 8 & Single - 1 \\
\hline \multirow{3}{*}{ Ulcer } & Single - 3 & Long segment - 10 & $1 / 4-1 / 2-12$ \\
\hline & Few - 5 & Whole tertile - 15 & $>1 / 2-18$ \\
\hline & Multiple - 10 & & \\
\hline \multicolumn{4}{|l|}{ Third tertile } \\
\hline \multirow[t]{3}{*}{ Villous appearance } & Normal - 0 & Short segment - 8 & Single - 1 \\
\hline & Edematous - 1 & Long segment - 12 & Patchy - 14 \\
\hline & & Whole tertile - 20 & Diffuse - 17 \\
\hline \multirow[t]{2}{*}{ Ulcer } & None - 0 & Short segment - 5 & $<1 / 4-9$ \\
\hline & Single -3 & Long segment - 10 & $1 / 4-1 / 2-12$ \\
\hline Stenosis & Multiple - 20 & & \\
\hline
\end{tabular}

Adapted from Gralnek IM, et al. Aliment Pharmacol Ther 2008;27:146-154, with permission from John Wiley and Sons. ${ }^{19}$

assessment using CE predicted subsequent flares within 6 months in asymptomatic patients. A study by Oliva et al. ${ }^{24}$ found that the application of the pan-enteric CE for detecting mucosal healing in pediatric patients led to important modifications in therapy. At present, there is insufficient evidence to recommend routine CE following a normal MRE or CTE to assess for mucosal healing, but this may evolve as an important indication in the near future.

The previously mentioned LS (section 1.1) can be used to objectively assess disease severity. Another simpler scoring system, the capsule endoscopy Crohn's disease activity index (CECDAI), has also been specifically developed for CD (Table
$2)^{25}$ A recent study comparing the 2 scores found that LS 135 and 790 were equivalent to CECDAI values of 4.9 and 6.9, respectively. There was a strong correlation between the 2 scores, but the CECDAI was more reflective of extensive inflammation and high clinical activity. ${ }^{26}$ Another study also found a strong correlation between the 2 scores, but only moderate correlation with fecal calprotectin (FC) ${ }^{27}$ However, there is no gold standard or widely accepted best scoring system in practice as of now.

As mentioned previously, the use of PCs is strongly recommended in cases of established CD given the high risk of strictures, even if cross-sectional imaging such as MRE or CTE is 
Table 2. CECDAI Scoring System

\begin{tabular}{|c|c|c|}
\hline CECDAI & Proximal & Distal \\
\hline \multicolumn{3}{|l|}{ A. Inflammation score } \\
\hline \multicolumn{3}{|l|}{$0=$ None } \\
\hline \multicolumn{3}{|c|}{$2=$ Severe edema $/$ hyperemia/denudation } \\
\hline \multicolumn{3}{|c|}{$3=$ Bleeding, exudate, aphthae, erosion, small ulcer $(\geq 0.5 \mathrm{~cm})$} \\
\hline \multicolumn{3}{|l|}{$5=$ Large ulcer $(2 \mathrm{~cm})$} \\
\hline \multicolumn{3}{|l|}{ B. Extent of disease score } \\
\hline \multicolumn{3}{|l|}{$0=$ None } \\
\hline \multicolumn{3}{|l|}{$1=$ Focal disease (single segment) } \\
\hline \multicolumn{3}{|l|}{$0=$ None } \\
\hline \multicolumn{3}{|l|}{$1=$ Single-passed } \\
\hline \multicolumn{3}{|l|}{2 = Multiple-passed } \\
\hline \multicolumn{3}{|l|}{ Segmental score $=A \times B+C$} \\
\hline Total score $=(\mathrm{A} 1 \times \mathrm{B} 1+\mathrm{C} 1)+(\mathrm{A} 2 \times \mathrm{B} 2+\mathrm{C} 2)$ & & \\
\hline
\end{tabular}

CECDAl, capsule endoscopy Crohn's disease activity index.

Adapted from Gal E, et al. Dig Dis Sci 2008;53:1933-1937, with permission from Springer Nature. ${ }^{25}$

normal.

Although the data is more limited compared to UC, CCE can also be used to assess disease activity for colonic $\mathrm{CD}^{28}$

\section{To Assess Postoperative Recurrence of CD Following Ileocolonic Resection}

Postoperative recurrence of $\mathrm{CD}$ following a limited right hemicolectomy occurs in up to $70 \%$ of cases, ${ }^{29}$ and recent studies have shown that early detection of endoscopic recurrence is important for starting biological therapy and the prevention of clinical recurrence and repeat surgery. ${ }^{30}$ Although the standard method for assessing this is ileocolonoscopy, studies are emerging on the indication of CCE. One of the earliest studies by Pons Beltran, ${ }^{31}$ demonstrated that CE detected CD recurrence in 15/22 (62\%) patients, whereas ileocolonoscopy detected inflammatory lesions in only 6/24 (25\%) patients (all in the neoterminal ileum). Similarly, in another study, CE was shown to detect lesions outside the scope of ileocolonoscopy in two-thirds of patients. ${ }^{32}$ The most recent study by Sorrentino et al. ${ }^{33}$ found similar findings which led to an upgrade of management in 12 out of 23 patients (52\%). Therefore, monitoring small bowel lesions with CE, which is beyond the scope of conventional ileocolonoscopy, may be beneficial in improving postoperative outcomes in CD.

\section{To Monitor Disease Activity or Mucosal Healing of the Colon in UC}

Many patients with IBD would prefer noninvasive methods of assessments, and it is justifiable that the indications for CCE-2 have quickly expanded from general colorectal cancer screening to include surveillance in IBD. A study by Hosoe et al. ${ }^{34}$ showed that there was a reasonably good correlation between CCE-2 and conventional colonoscopy. Okabayashi et al. ${ }^{35}$ proposed a 1-day simple regimen of CCE-2 optimized for UC to achieve better acceptance where $500 \mathrm{~mL}$ of hypertonic polyethylene glycol solution, followed by $250 \mathrm{~mL}$ of water, is ingested 2.5 hours before, and then 1, 3, and 6 hours after capsule ingestion until its excretion; castor oil is added to the second booster. Following this, a capsule score for UC (CSUC) was developed to objectively assess disease severity in UC using CCE-2 (Table 3). ${ }^{36}$ Correlation between CCE-2 findings and the risk of relapse has also been reported. ${ }^{37}$

Although it is unlikely that CCE will replace fecal biomarkers and ileocolonoscopy as the first-line investigation for UC, 
Table 3. Capsule Scoring of Ulcerative Colitis (CSUC)

\begin{tabular}{lll}
\hline Descriptor (score most severe lesions) & Likert Scale Anchor (points) & \multicolumn{1}{c}{ Definition } \\
\hline Vascular pattern & Normal (0) & Normal vascular pattern \\
& Patchy obliteration (1) & Obliterated area $\leq 30 \%$ \\
& Obliterated (2) & Obliterated area $>30 \%$ \\
Bleeding & None (0) & No visible blood detected by SBI \\
& Mild (1) & No. bleeding picture detected by SBI $\leq 10$ \\
Erosions and ulcers & Severe (2) & No. bleeding picture detected by SBI $>10$ \\
& None (0) & Normal mucosa, no visible erosions or ulcers \\
& Erosions (1) & Tiny ( $\leq 5 \mathrm{~mm}$ ) defects in the mucosa \\
& Superficial ulcer (2) & Larger ( $>5 \mathrm{~mm}$ ) defects in the mucosa \\
& Deep ulcer (3) & Larger ( $>5 \mathrm{~mm}$ ) and deeper excavated defects in the mucosa, \\
\hline
\end{tabular}

SBI, suspected blood indicator.

Adapted from Hosoe N, et al. Inflamm Bowel Dis 2018;24:2641-2647, with permission from Oxford University Press. ${ }^{36}$

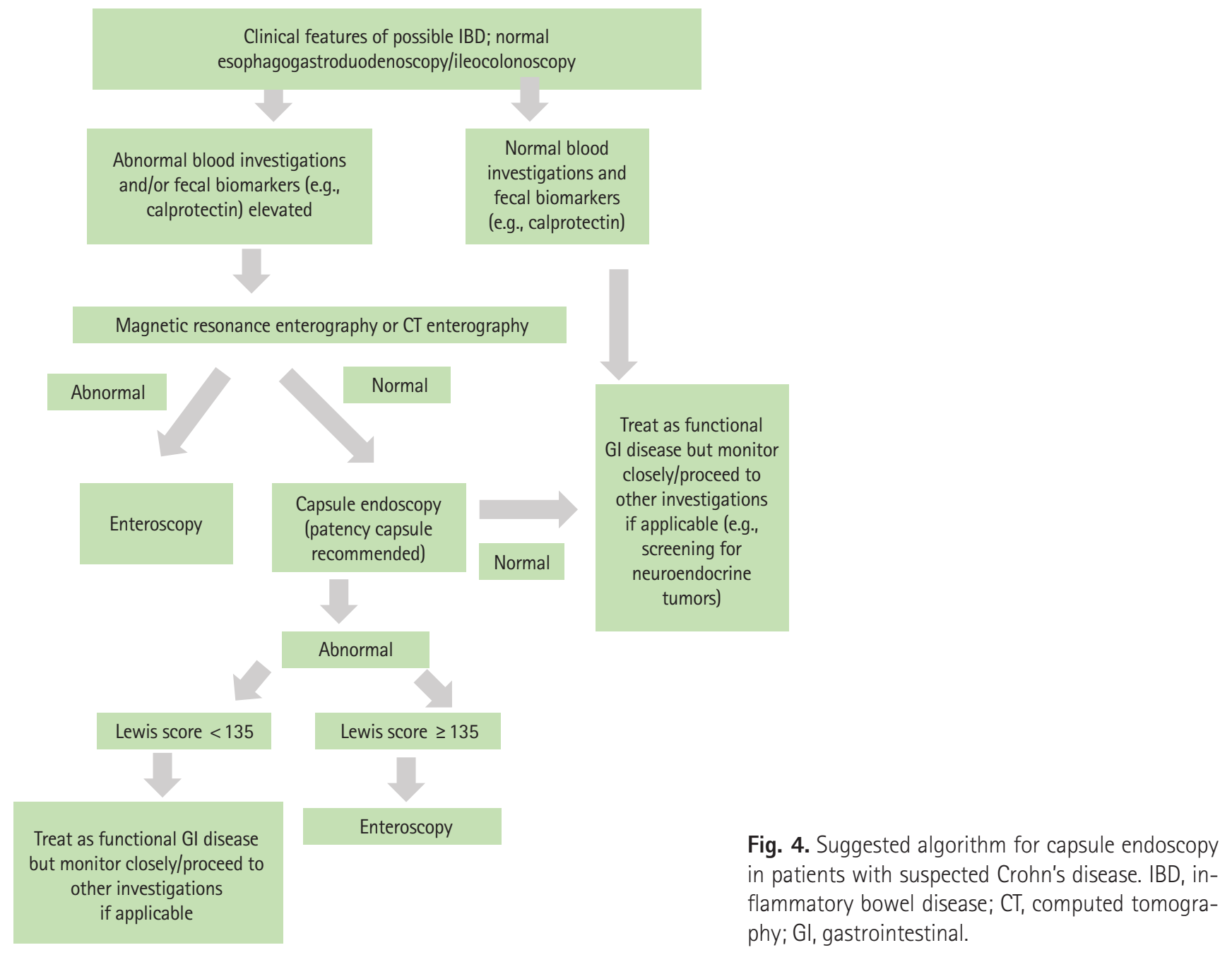


it remains a viable alternative; for example, in those with indeterminate results of fecal biomarkers, but who are reluctant or at high risk for ileocolonoscopy.

\section{SUGGESTED ALGORITHM FOR CAPSULE ENDOSCOPY IN IBD}

\section{Capsule Endoscopy (SBCE) in Suspected Small Bowel CD}

In patients with clinically suspected IBD (in particular isolated $\mathrm{CD}$ ), $\mathrm{FC}$ is probably the most cost-effective first investigation. A meta-analysis by Kopylov et al. ${ }^{38}$ found the sensitivity and specificity of FC to be 0.89 and 0.55 , respectively, if a cut of $>50 \mu \mathrm{g} / \mathrm{g}$ was used, and a recent study by Shimoyama et al. ${ }^{39}$ also concluded that using fecal biomarkers was the most costeffective method for screening for small bowel pathology such as $\mathrm{CD}$. If FC is elevated, we suggest MRE (or CTE if MRE is not readily available) as the first investigation. If MRE or CTE is abnormal, enteroscopy rather than $\mathrm{CE}$ is recommended given the risk of capsule retention. If the MRE or CTE is normal, CE should be considered if there is a strong clinical suspicion of CD based on symptoms and other biomarkers. The application of a scoring system may be useful for objective assessment of the need for further workup. It may be prudent to consider a PC in all cases of suspected IBD although the overall risk of capsule retention is low. The algorithm for CE for suspected small bowel CD is illustrated in Fig. 4.

\section{Capsule Endoscopy in Established CD}

In patients with confirmed CD, MRE, or CTE is usually adequate to assess small bowel involvement. In cases where MRE or CTE is abnormal, enteroscopy is recommended if biopsies and further assessment are required. However, as mentioned previously, SBCE may be considered after a normal MRE or CTE in cases of severe malnutrition, ongoing symptoms, and unexplained anemia. As the goalpost of therapy has shifted, SBCE may be considered for a full assessment of mucosal healing throughout the small bowel. The objective assessment of disease activity can be carried out using the LS or the CECDAI.

PCs are recommended in cases of established IBD given the high risk of capsule retention.

Similarly, CCE-2 may be a useful adjunct or alternative for assessing postoperative $\mathrm{CD}$ recurrence in the neoterminal ileum following ileocolonic resection and monitoring of colonic $\mathrm{CD}$. The PCC is advantageous for assessing CD patients with extensive small bowel and colonic involvement. The system platform and software use a novel assessment methodology for documenting disease extent and severity.

However, incorporating CE as a standard investigation in all cases of CD may not be practical in many countries. CE is expensive and the addition of PCs will only add to the cost. Biological therapy also remains unaffordable to many patients in these countries, and the identification of mild mucosal inflammation in the small bowel may not lead to change in management as in the more developed nations. Furthermore, the LS, CECDAI, and CSUC may be difficult or cumbersome to calculate in daily clinical practice. Another disadvantage is that it is time-consuming. However, several methods for creating automated software for ulcer detection ${ }^{40}$ have been developed, and hopefully in future will also cover typical endoscopic features of inflammation. The algorithm for CE for confirmed CD is illustrated in Fig. 5.

\section{Capsule Endoscopy (CCE) in UC}

For monitoring disease activity of UC, most physicians would still prefer conventional colonoscopy, especially for enhancing the detection of dysplastic lesions with chromoendoscopy or if therapeutic intervention is considered (e.g., dilatation of

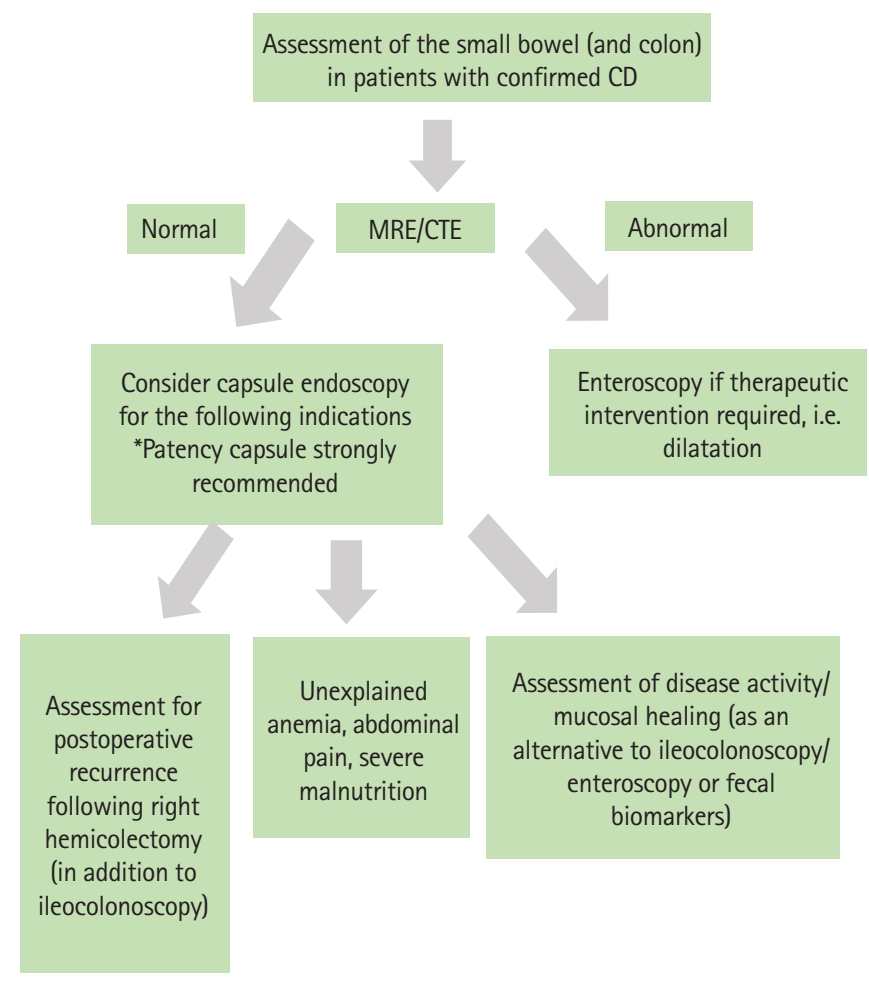

Fig. 5. Suggested algorithm for capsule endoscopy in patients with confirmed Crohn's disease (CD). MRE, magnetic resonance enterography; CTE, computed tomography enterography. 


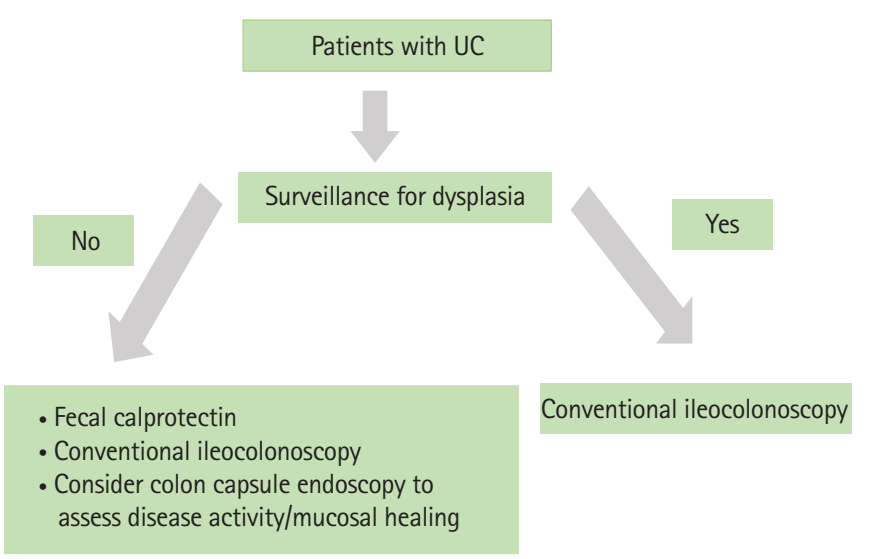

Fig. 6. Suggested algorithm for capsule endoscopy in patients with ulcerative colitis (UC).

stricture). However, CE is an attractive noninvasive method for disease monitoring, which include assessments of mucosal healing in patients in clinical remission, and may be considered in this setting. The CSUC can be used for standardized and objective assessments of disease activity in this setting, but further studies on this are required. The algorithm for CE in UC is illustrated in Fig. 6.

\section{CONCLUSION}

CE has revolutionized noninvasive imaging of the small bowel and colon. Current and future developments such as magnetic CE, which allows real-time maneuverability of the capsule, ${ }^{41}$ automated software for detection of ulcer/inflammation, and further development of designated IBD systems will almost certainly enhance its role in the diagnosis and monitoring of IBD.

\section{FINANCIAL SUPPORT}

The authors received no financial support for the research, authorship, and/or publication of this article.

\section{CONFLICT OF INTEREST}

No potential conflict of interest relevant to this article was reported.

\section{AUTHOR CONTRIBUTION}

Writing of original draft: Hilmi I. Writing, review, and editing:
Kobayashi T. Approval of final manuscript: all authors.

\section{ORCID}

Hilmi I

Kobayashi T

https://orcid.org/0000-0001-7091-0032

https://orcid.org/0000-0002-2073-4234

\section{REFERENCES}

1. Iddan G, Meron G, Glukhovsky A, Swain P. Wireless capsule endoscopy. Nature 2000;405:417.

2. Eliakim R, Fireman Z, Gralnek IM, et al. Evaluation of the PillCam colon capsule in the detection of colonic pathology: results of the first multicenter, prospective, comparative study. Endoscopy 2006;38:963-970.

3. Eliakim R, Spada C, Lapidus A, et al. Evaluation of a new panenteric video capsule endoscopy system in patients with suspected or established inflammatory bowel disease: feasibility study. Endosc Int Open 2018;6:E1235-E1246.

4. Cosnes J, Gower-Rousseau C, Seksik P, Cortot A. Epidemiology and natural history of inflammatory bowel diseases. Gastroenterology 2011;140:1785-1794.

5. Dionisio PM, Gurudu SR, Leighton JA, et al. Capsule endoscopy has a significantly higher diagnostic yield in patients with suspected and established small-bowel Crohn's disease: a meta-analysis. Am J Gastroenterol 2010;105:1240-1248.

6. Kopylov U, Yung DE, Engel T, et al. Diagnostic yield of capsule endoscopy versus magnetic resonance enterography and small bowel contrast ultrasound in the evaluation of small bowel Crohn's disease: systematic review and meta-analysis. Dig Liver Dis 2017;49:854-863.

7. González-Suárez B, Rodriguez S, Ricart E, et al. Comparison of capsule endoscopy and magnetic resonance enterography for the assessment of small bowel lesions in Crohn's disease. Inflamm Bowel Dis 2018;24:775-780.

8. Shim KN, Jeon SR, Jang HJ, et al. Quality indicators for small bowel capsule endoscopy. Clin Endosc 2017;50:148-160.

9. Enns RA, Hookey L, Armstrong D, et al. Clinical practice guidelines for the use of video capsule endoscopy. Gastroenterology 2017;152:497-514.

10. Ooi CJ, Makharia GK, Hilmi I, et al. Asia Pacific Consensus Statements on Crohn's disease. Part 1: definition, diagnosis, and epidemiology. (Asia Pacific Crohn's Disease Consensus: Part 1). J Gastroenterol Hepatol 2016;31:45-55.

11. Lim YJ, Lee OY, Jeen YT, et al. Indications for detection, completion, and retention rates of small bowel capsule endoscopy 
based on the 10-year data from the Korean Capsule Endoscopy Registry. Clin Endosc 2015;48:399-404.

12. Rezapour M, Amadi C, Gerson LB. Retention associated with video capsule endoscopy: systematic review and meta-analysis. Gastrointest Endosc 2017;85:1157-1168.

13. Fernández-Urién I, Carretero C, González B, et al. Incidence, clinical outcomes, and therapeutic approaches of capsule endoscopy-related adverse events in a large study population. Rev Esp Enferm Dig 2015;107:745-752.

14. Liao Z, Gao R, Xu C, Li ZS. Indications and detection, completion, and retention rates of small-bowel capsule endoscopy: a systematic review. Gastrointest Endosc 2010;71:280-286.

15. Caunedo-Alvarez A, Romero-Vazquez J, Herrerias-Gutierrez JM. Patency and Agile capsules. World J Gastroenterol 2008; 14:5269-5273.

16. Kopylov U, Nemeth A, Cebrian A, et al. Symptomatic retention of the patency capsule: a multicenter real life case series. Endosc Int Open 2016;4:E964-E969.

17. Lee HS, Lim YJ, Kim KO, et al. Outcomes and management strategies for capsule retention: a Korean Capsule Endoscopy Nationwide Database Registry study. Dig Dis Sci 2019;64: 3240-3246

18. Nemeth A, Wurm Johansson G, Nielsen J, Thorlacius H, Toth E. Capsule retention related to small bowel capsule endoscopy: a large European single-center 10-year clinical experience. United European Gastroenterol J 2017;5:677-686.

19. Gralnek IM, Defranchis R, Seidman E, Leighton JA, Legnani P, Lewis BS. Development of a capsule endoscopy scoring index for small bowel mucosal inflammatory change. Aliment Pharmacol Ther 2008;27:146-154.

20. Monteiro S, Boal Carvalho P, Dias de Castro F, et al. Capsule endoscopy: diagnostic accuracy of Lewis score in patients with suspected Crohn's disease. Inflamm Bowel Dis 2015; 21:2241-2246.

21. Dubcenco E, Jeejeebhoy KN, Petroniene R, et al. Capsule endoscopy findings in patients with established and suspected small-bowel Crohn's disease: correlation with radiologic, endoscopic, and histologic findings. Gastrointest Endosc 2005;62:538-544.

22. Dussault C, Gower-Rousseau C, Salleron J, et al. Small bowel capsule endoscopy for management of Crohn's disease: a retrospective tertiary care centre experience. Dig Liver Dis 2013;45:558-561.

23. Ben-Horin S, Lahat A, Amitai MM, et al. Assessment of small bowel mucosal healing by video capsule endoscopy for the prediction of short-term and long-term risk of Crohn's disease flare: a prospective cohort study. Lancet Gastroenterol Hepatol 2019;4:519-528

24. Oliva S, Aloi M, Viola F, et al. A treat to target strategy using panenteric capsule endoscopy in pediatric patients with Crohn's disease. Clin Gastroenterol Hepatol 2019;17:20602067.

25. Gal E, Geller A, Fraser G, Levi Z, Niv Y. Assessment and validation of the new capsule endoscopy Crohn's disease activity index (CECDAI). Dig Dis Sci 2008;53:1933-1937.

26. Omori T, Kambayashi H, Murasugi S, et al. Comparison of Lewis score and capsule endoscopy Crohn's disease activity index in patients with Crohn's disease. Dig Dis Sci 2020;65: 1180-1188.

27. Yablecovitch D, Lahat A, Neuman S, et al. The Lewis score or the capsule endoscopy Crohn's disease activity index: which one is better for the assessment of small bowel inflammation in established Crohn's disease? Therap Adv Gastroenterol 2018;11:1756283X17747780.

28. D'Haens G, Löwenberg M, Samaan MA, et al. Safety and feasibility of using the second-generation PillCam colon capsule to assess active colonic Crohn's disease. Clin Gastroenterol Hepatol 2015;13:1480-1486.

29. Rutgeerts P, Geboes K, Vantrappen G, Beyls J, Kerremans R, Hiele M. Predictability of the postoperative course of Crohn's disease. Gastroenterology 1990;99:956-963.

30. De Cruz P, Kamm MA, Hamilton AL, et al. Crohn's disease management after intestinal resection: a randomised trial. Lancet 2015;385:1406-1417.

31. Pons Beltrán V, Nos P, Bastida G, et al. Evaluation of postsurgical recurrence in Crohn's disease: a new indication for capsule endoscopy? Gastrointest Endosc 2007;66:533-540.

32. Bourreille A, Jarry M, D'Halluin PN, et al. Wireless capsule endoscopy versus ileocolonoscopy for the diagnosis of postoperative recurrence of Crohn's disease: a prospective study. Gut 2006;55:978-983.

33. Sorrentino D, Nguyen VQ. Clinically significant small bowel Crohn's disease might only be detected by capsule endoscopy. Inflamm Bowel Dis 2018;24:1566-1574.

34. Hosoe N, Matsuoka K, Naganuma M, et al. Applicability of second-generation colon capsule endoscope to ulcerative colitis: a clinical feasibility study. J Gastroenterol Hepatol 2013;28:1174-1179.

35. Okabayashi S, Kobayashi T, Nakano M, et al. A simple 1-day colon capsule endoscopy procedure demonstrated to be a highly acceptable monitoring tool for ulcerative colitis. Inflamm Bowel Dis 2018;24:2404-2412. 
36. Hosoe N, Nakano M, Takeuchi K, et al. Establishment of a novel scoring system for colon capsule endoscopy to assess the severity of ulcerative colitis-capsule scoring of ulcerative colitis. Inflamm Bowel Dis 2018;24:2641-2647.

37. Takano R, Osawa S, Uotani T, et al. Evaluating mucosal healing using colon capsule endoscopy predicts outcome in patients with ulcerative colitis in clinical remission. World J Clin Cases 2018;6:952-960.

38. Kopylov U, Yung DE, Engel T, et al. Fecal calprotectin for the prediction of small-bowel Crohn's disease by capsule endoscopy: a systematic review and meta-analysis. Eur J Gastroen- terol Hepatol 2016;28:1137-1144.

39. Shimoyama T, Yamamoto T, Umegae S, Matsumoto K. Faecal biomarkers for screening small bowel inflammation in patients with Crohn's disease: a prospective study. Therap Adv Gastroenterol 2017;10:577-587.

40. Klang E, Barash Y, Margalit RY, et al. Deep learning algorithms for automated detection of Crohn's disease ulcers by video capsule endoscopy. Gastrointest Endosc 2020;91:606-613.

41. Carpi F. Magnetic capsule endoscopy: the future is around the corner. Expert Rev Med Devices 2010;7:161-164. 